\title{
Wide-Area Protection and Power System Utilization
}

\author{
JOACHIM BERTSCH, MEMBER, IEEE, CÉDRIC CARNAL, \\ DANIEL KARLSSON, SENIOR MEMBER, IEEE, JOHN MCDANIEL, AND KHOI VU
}

\section{Invited Paper}

This paper describes basic principles and philosophy for wide-area protection schemes, also known as remedial action schemes (RAS) or system protection schemes (SPS). In the areas of power system automation and substation automation, there are two parallel trends in different directions: centralization and decentralization. More and more functions are moved from local and regional control centers toward the central or national control center. At the same time we also observe more and more "intelligence" and "decision-power" moving closer toward the actual power system process. We also see a great deal of functional integration, i.e., more and more functionality enclosed in the same hardware. This raises discussions concerning reliability (security and dependability). The main targets for this paper is therefore to: 1) sort out the terminology used in this area; 2) describe different application areas and related requirements; 3) illustrate different design principles- “top-down," "bottom-up," hierarchy, flat, etc., for different applications; and 4) identify similarities and differences between classic equipment protection and system protection-concerning philosophy as well as concerning product and system design.

The theme of the paper is on the use of information technology to obtain more flexibility and smartness in power-system controls.

Keywords-Architecture, energy management system (EMS), global positioning system (GPS), phasor measurement unit (PMU), supervisory control and data acquisition (SCADA), system protection scheme (SPS), system protection terminal, wide-area measurement system (WAMS).

\section{NOMENCLATURE}

WAMS Wide-Area Measurement System.

Manuscript received July 29, 2003; revised September 5, 2003.

J. Bertsch and C. Carnal are with Power Technology Systems, ABB Automation, Baden CH-5401, Switzerland (e-mail: Cedric.Carnal@ ch.abb.com).

D. Karlsson is with ABB Automation, Malmo SE-216 20, Sweden (e-mail: daniel.karlsson@gothiapower.com).

J. McDaniel is with ABB Automation, Sugar Land, TX USA (e-mail: John.McDaniel@us.abb.com).

$\mathrm{K} . \mathrm{Vu}$ is with ABB Corporate Research, Raleigh, NC USA (e-mail: Khoi.Vu@us.abb.com).

Digital Object Identifier 10.1109/JPROC.2005.847266
$\mathrm{HVdc}$
High-voltage dc.
AGC Automatic generation control.
SCADA Supervisory control and data acquisition.
EMS Energy management systems.
PMU Phasor measurement unit.
GPS Global postitioning system.
SVC Static var compensator.
EMC Electromagnetic compatibility.

\section{BACKGROUND AND INTRODUCTION}

There are a few basic facts and technological developments that have pushed the utility needs and the vendors' offers in wide-area protection and control.

1) The deregulated electricity market causes rather quick changes in the operational conditions. New, unknown, load flow patterns show up more frequently for the system operator.

2) Economic pressure on the electricity market and on grid operators forces them to maximize the utilization of high-voltage equipment, which very often means operation closer to the limits of the system and its components. For the same reason, there is also a wish to "push" the limits.

3) Reliable electricity supply is continually becoming more and more essential for the society and blackouts are becoming more and more costly whenever they occur.

4) Technical developments in communication technology and measurement synchronization, e.g., for reliable voltage phasor measurements, have made the design of system wide protection solutions possible. The use of phasor measurements also provides new possibilities for state estimator functions. Their main use so far has been for WAMS applications. 
5) There is a general trend to include both normal operation issues and disturbance handling into power system automation (PSA).

6) Wide-area disturbances during the last decades have forced/encouraged power companies to design system protection schemes to counteract voltage instability, angular instability, frequency instability, to improve damping properties or for other specific purposes, e.g., to avoid cascaded line trip.

7) A lot of research and development within universities and industry have significantly increased our knowledge about the power system phenomena causing widespread blackouts. Methods to counteract them have been or are being developed, e.g., [1], [2].

8) There is a heightened concern for security of power grids due to acts of coordinated sabotage, which traditionally were not considered in grid planning. Fast and efficient controls/protections are needed to stop the disruption from spreading.

\section{Market Demands ANd Utility NeEdS}

Based on the situation and new technology described above, grid companies can benefit a lot. Wide-area protection and emergency control systems can be introduced in the power system, either:

— to increase the power system transmission capability; or

- to increase the power system reliability; or

- a combination of the two.

This means that the grid company can extend the system operational limits, i.e., operate the system closer to the physical constraints, without any investments in high voltage equipment. The operational criteria will change from:

- "the power system should withstand the most severe credible contingency" to

- "the power system should withstand the most severe credible contingency, followed by protective actions from the wide-area protection system."

Certain power systems do not fulfill the design and operational criteria, which are based on the reliability requirements on the system, without installation of wide-area protection systems. The reliability requirements on such wide-area protection systems-dependability as well as security—are extremely high.

\section{A. How to Meet Present and Future Requirements}

Utility companies may require one package for the entire operation of the power system, including planning, operation, control, and protection-optimized on a "system" level, based on maximum profit and supply reliability. This "package" should consist of a number of integrated product offerings and should be compatible with present equipment. This approach benefits both retrofit and new installations. To allow for retrofit, such a package should use present technology and move toward standardized communication, standard input and output format, etc.
Large utilities, such as Hydro-Québec, BC Hydro, and Bonneville Power Administration, still design their own system protection schemes and defense plans, based on standard components available today. To make the area of system protection available also for other utilities, there is a market need for dedicated system protection devices and basic system protection design structures. Such systems could be based on standardized system protection terminals, with customized functionality, tied together in a wide-area protection system. Standardization, modularization and flexibility are important features in such a design.

\section{Key Concepts in PRotection AND CONTROL}

Protection is very closely related to circuit-breaker trip signals to disconnect faulty or overloaded equipment from the network, to save the component, and to reestablish normal operation of the healthy part of the power system and thereby continue electricity supply to the customers. Protection equipment is also aimed at protecting people, animals, and property from injury and damage due to electric faults. The situation when a protection device triggers is so severe that if the equipment is not tripped, it will be severely damaged or the surroundings will be exposed to serious danger.

System protection or wide-area protection is used to save the system from a partial or total blackout or brownout in operational situations when no particular equipment is faulted or operated outside its limitations. This situation could appear after the clearance of a very severe disturbance in a stressed operation situation or after an extreme load growth. Since it is a protection system, it will operate in such operational situations when the power system would break down if no protective actions were taken. Such protective actions also comprise shifts of setting groups and parameter values for different protection and control devices, blocking of tapchangers, switch in of shunt capacitors, etc.

Emergency control is associated with continuous control actions in order to save the power system, such as boosting the exciter on a synchronous generator or changing the power direction of an HVdc link.

Normal control actions are associated with continuous control activities, that can be either step-wise, e.g., tap-changer and shunt device, or continuous, such as frequency control. Normal control is preventive, i.e., actions are taken to adjust the power system operational conditions to the present and near future expected situation. Normal control is usually automatic, e.g., tap-changer, reactive shunt device, frequency control, and AGC.

The difference between normal and emergency control is the consequence for the power system if the control action is not performed. If a normal, preventive, control action is not performed, there is an increased risk for the loss of power system stability, i.e., stability will be lost if a severe disturbance occurs. If an emergency, corrective, control action is not performed, the system will go unstable. The response requirements (time and reliability) are normally higher for emergency control actions than for normal control actions. 
Emergency control functions are almost always automatic, while normal control actions can be either automatic or manual, e.g., in conjunction with alarms. The actions taken in the power system are, however, quite similar for both normal control and emergency control. Protection, system protection and emergency control comprise corrective (or curative) measures, i.e., actions are really needed to save the component or the system. Protection could very well be regarded as binary (on/off) emergency control, but by tradition, protection is quite specific.

Voltage control comprises actions like AVR, tap-changers and shunt devices, automatic or manual control. The control variable is usually the voltage level or reactive power flow. For system protection purposes or "emergency control," also load shedding and AGC can be used.

Primary frequency control is normally performed in the power stations by the governor controls, while secondary frequency control is performed by the AGC change of set-point or start/stop of units by the dispatcher. For emergency control, load shedding as well as actions that reduces the voltage, and hence the voltage sensitive part of the load, can be used.

Automatic power flow control is performed by AGC, $\mathrm{HVdc}$ control, united power flow controller (UPFC), thyristor-controlled series capacitor (TCSC), phase-shift transformers, etc. Load level, network topology, and generation dispatch are the most common parameters that influence the power flow.

Angle control is more accurate if based on PMUs. Without PMUs, power flow is an indirect method of measuring and controlling the angle. The actions are similar as for power flow control.

$S C A D A / E M S$ functions are tools that assist the power system/grid operator in his effort to optimize the power system operation, with respect to economy, operational security, and robustness, as well as human and material safety. Operator actions are normally (at least supposed to be) preventive, i.e., actions taken to adjust the power system operational conditions to the present and near future expected situation. Preventive actions, based on simple criteria, can beneficially be implemented in SCADA/EMS and be performed automatically or be suggested and then released or blocked by the operator. SCADA/EMS systems are normally to slow to capture power system dynamics.

The $P M U$ is a device for synchronized measurement of ac voltages and currents, with a common time (angle) reference. The most common time reference is the GPS signal, which has a precision down to $1 \mu \mathrm{s}$. In this way, the ac quantities can be time-stamped and measured as complex values and represented by their magnitude and phase angle.

\section{Power System Phenomena to COUNTERACT}

Utility needs and problems are often formulated in very loose terms, such as "intelligent load shedding," "protection system against major disturbances," and "counteract cascaded line tripping." These needs have to be broken down to physical phenomena, such as protection against:
- transient angle instability (first swing);

- small signal angle instability (damping);

- frequency instability;

- short-term voltage instability;

- long-term voltage instability;

- cascading outages.

\section{A. Transient Angle Instability}

Offline design studies have normally been made to ensure the transient angle stability for credible contingencies. Parameters which are required to be influenced during the design stage are line circuit impedance, trip time, autoreclosing, inertia constants, and additional equipment such as series capacitors and breaking resistors. In the operational stage, certain power flow levels must not be exceeded. PMUs allow for direct and fast angle measurement, instead of indirect power measurement, and more accurate control algorithms for emergency control or protective actions can be designed.

\section{B. Small Signal Angle Instability}

Offline studies, such as eigenfrequency analysis and time simulations, have to be done to check the damping conditions for different frequencies. SVCs in the network and power system stabilizers on the generators are common means to counteract power oscillations. Again, PMUs in the power system can provide accurate angle measurements.

\section{Frequency Instability}

Frequency instability is most commonly the result of a sudden, large generation deficit. Automatic underfrequency controlled load shedding is the widely used measure to counteract a system breakdown in such situations. Also, the time derivative of the frequency is used in some applications. In case of overfrequency, due to sudden loss of load, generators can be shed.

\section{Short-Term Voltage Instability}

Short-term voltage instability is normally associated with an extremely severe reduction of the network capacity, e.g., caused by the trip of several parallel lines due to a bush fire. Characteristic for the short-term voltage instability is that there is no stable equilibrium point immediately after the clearance of the initial fault(s). Remedial actions to save the system in such a situation therefore have to be fast (a few seconds or fractions of a second) and powerful (e.g., large amount of load shedding).

\section{E. Long-Term Voltage Instability}

When a power system is in transition toward a "long-term voltage instability," the power system "survived" the initial disturbance, i.e., there was a stable equilibrium point immediately after the clearance of the disturbance. However, load recovery and tap-changer operation cause the transmission system voltage to decrease and the collapse occurs in the time scale of $10 \mathrm{~s}$ to $30 \mathrm{~min}$. Without any initial disturbance, a long-term voltage instability might occur due to a very large and rapid load increase. 


\section{Protection against Voltage Instability}

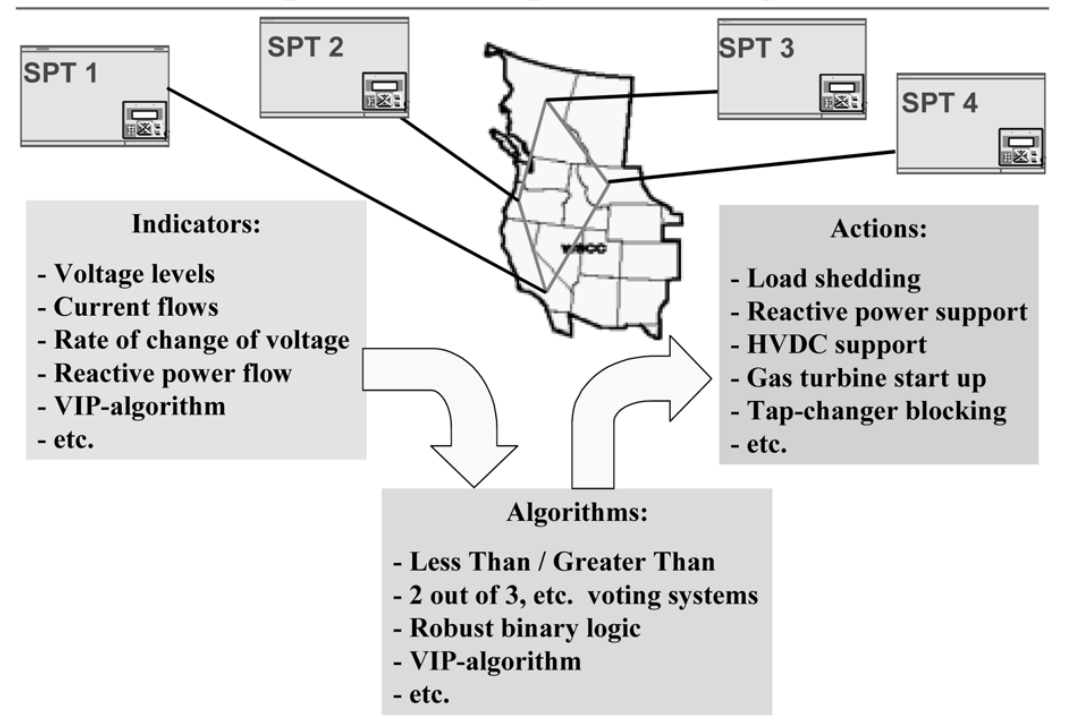

Fig. 1. Terminal-based wide-area protection system against voltage instability.

\section{F. Cascading Outages}

Cascading outages of lines or generators might have different origins, but are mainly associated with some kind of overload, followed by trip of one line or generator unit, which cause an increased overload on the remaining units, and so on. In such situations load shedding or generation rejection might be required to preserve the integrity of the power system.

For each phenomenon, a reliable (based on redundancy and robustness) protection system has to be designed, with respect to input variables, decision criteria, and output actions. Parallel systems counteracting different phenomena and different layers of safety nets can be designed and coordinated.

\section{REQUiREMENTS ON PROTECTION COMPARED to SCADA/EMS}

Products for wide-area protection and emergency control should be designed and manufactured in a similar way as conventional equipment protection, concerning standardization, flexibility, hardware and software modularization, configuration, and functionality. Maximum benefit from available hardware that have passed different kinds of EMC and environmental tests, as well as software functions available from other protection terminals, has to be made. Standardized communication protocols and hardware are also essential.

SCADA/EMS functions based on phasor measurements and inputs from system protection terminals should in general be compatible with present and future SCADA/EMS systems.

Based on synchronized phasor measurements, more efficient state estimation can be performed. Based on fast and reliable state estimation a variety of system stability indexes can be derived and monitored online to the system operator. Different (faster than real-time) stability programs for a number of contingencies can then be run to evaluate risks and margins.

Different kinds of "intelligent" load shedding can be ordered more or less automatically from the SCADA/EMS system in case of energy shortage on the electricity market or other limitations in the power system operation, that can be planned in advance. With access to wide-area measurements, such a system can be made adaptive to cope with the actual system conditions, such as load flow pattern and voltage levels.

It is necessary to distinguish between protection and SCADA/EMS, since this reflects the organizational structure of utility and grid company companies: the responsibilities for protection and for SCADA/EMS are given to different departments within the company.

\section{Possible Design ARchitectures}

Since the requirements for a wide-area protection system can vary from one utility company to another, the architecture for such a system must be designed according to what technologies the utility possesses at the given time. Also, to avoid becoming obsolete, the design must be chosen to fit the technology migration path that the utility in question will take. Three major design approaches are discussed below.

\section{A. Enhancements to SCADA/EMS}

At one end of the spectrum, enhancements to the existing EMS/SCADA can be made. These enhancements are aimed at two key areas: information availability and information interpretation. Simply put, if the operator has all vital information at his fingertips and good analysis facilities, he can operate the grid in an efficient way. For example, with better analysis tool for voltage instability, the operator can accurately track the power margin across an interface, and thus can confidently push the limit of transfer across that interface. 


\section{Hierarchical System Protection Architecture}

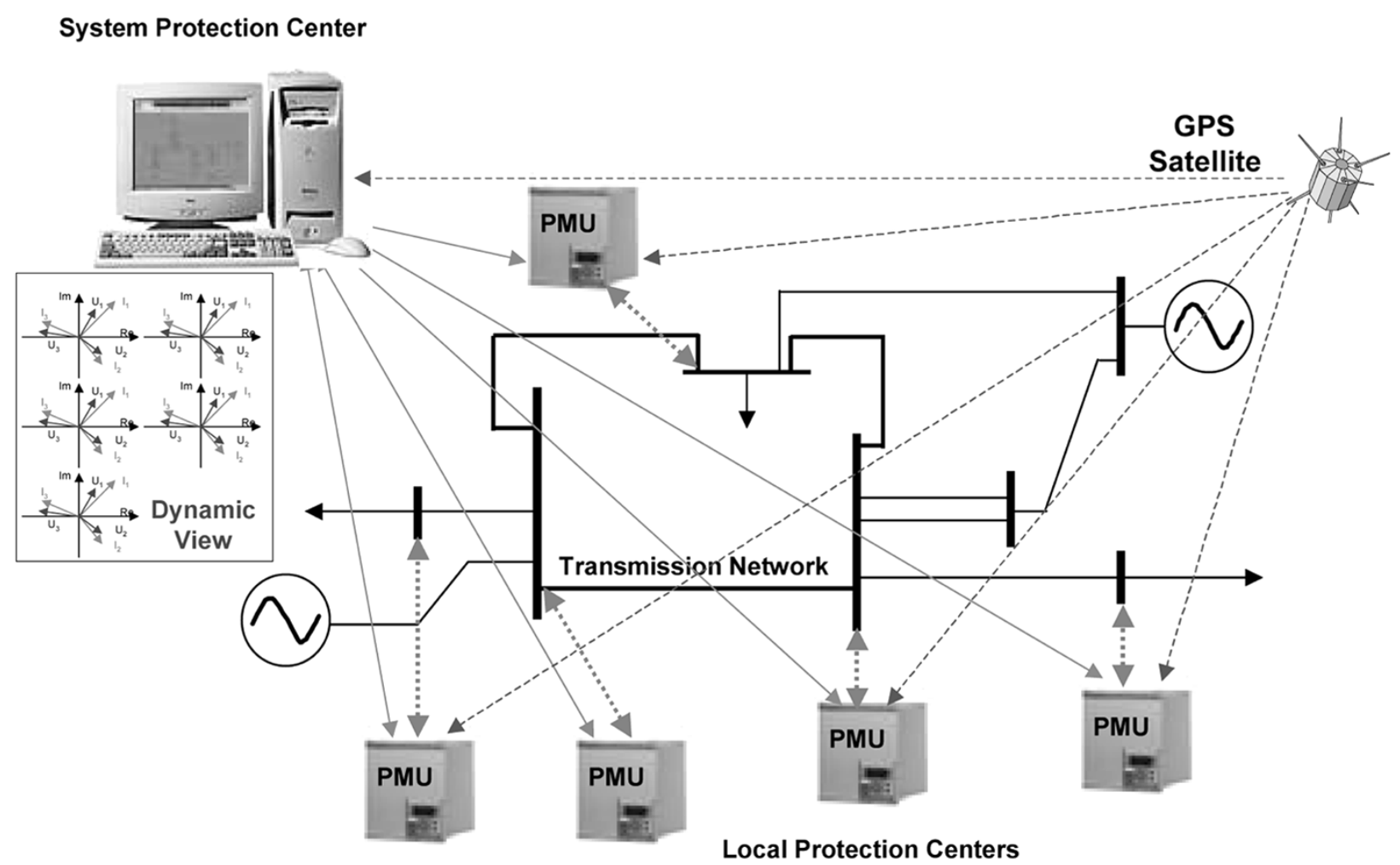

Fig. 2. Multilayered wide-area protection architecture.

SCADA/EMS system capability has greatly improved during recent years, due to improved communication facilities and highly extended data handling capability. New transducers such as the PMUs can provide time-synchronized measurements from all over the grid. Based on these measurements, improved state estimators can be derived.

Advanced algorithms and calculation programs that assist the operator can also be included in the SCADA system, such as "faster than real time simulations" to calculate power transfer margins based on contingencies.

The possibilities of extending the SCADA/EMS system with new functions tend to be limited. Therefore, it might be relevant to provide new SCADA/EMS functions as "stand alone" solutions, more or less independent of the ordinary SCADA/EMS system. Such functions could be load shedding, due to lack of generation or due to excessive market price.

\section{B. "Flat Architecture" With System Protection Terminals}

Protection devices or terminals are traditionally used in protecting equipment (lines, transformers, etc.). Modern protection devices have sufficient computing and communications capabilities that they are capable of performing beyond the traditional functions. When connected together via communications links, these devices can process intelligent algorithms (or "agents") based on data collected locally or shared with other devices.

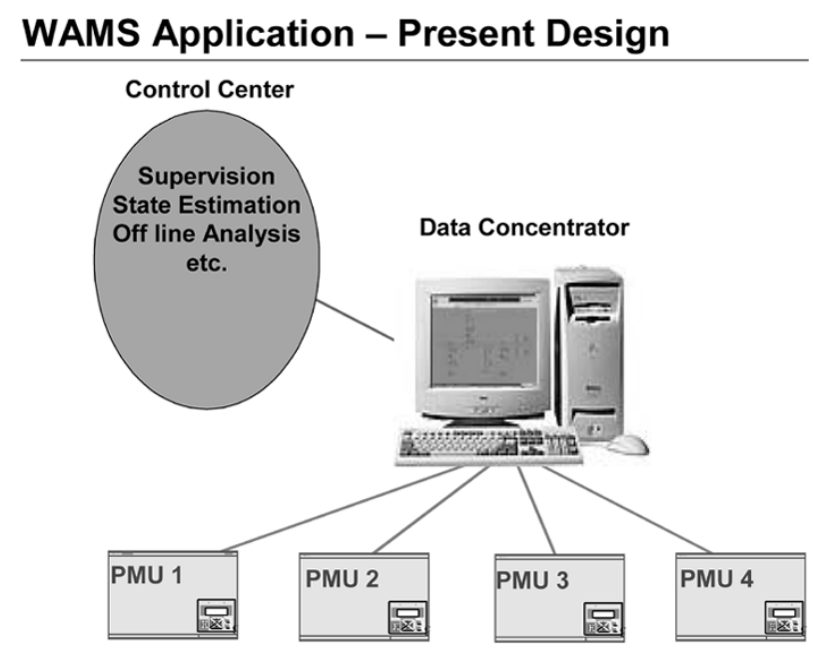

Fig. 3. WAMS design.

Powerful, reliable, sensitive, and robust, wide-area protection systems can be designed based on decentralized, especially developed interconnected system protection terminals. These terminals are installed in substations, where actions are to be made or measurements are to be taken. Actions are preferably local, i.e., transfer trips should be avoided, to increase security. Relevant power system variable data is transferred through the communication system that ties the terminals together. Different schemes, e.g., against voltage instability and against frequency instability, can be implemented in the same hardware. 


\section{WAMS \& Protection - Hub based Design}

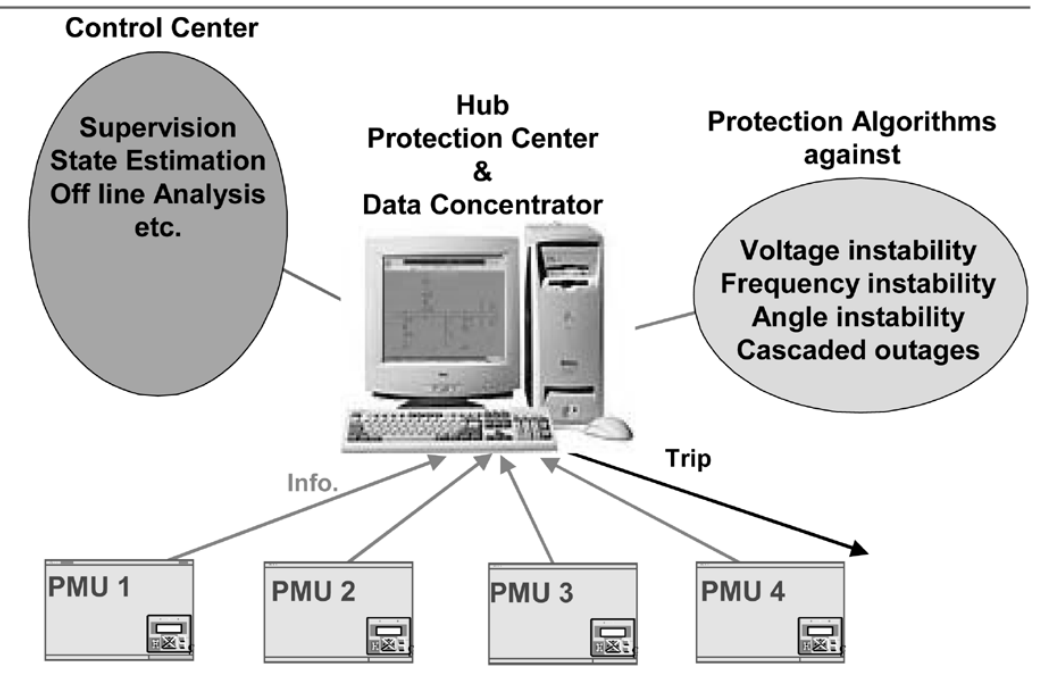

Fig. 4. Hub based wide-area protection design.

Different layers of protection can be used, compared with the different zones of a distance protection. The voltage is for example measured in eight $400-\mathrm{kV}$ nodes in a protection system against voltage instability. In a certain node, a certain action is taken if:

— six of the eight voltages are low (e.g., $<380 \mathrm{kV}$ ); or

- four of the eight voltages are very low (e.g., $<370 \mathrm{kV}$ ); or

- the local voltage is extremely low (e.g., $<360 \mathrm{kV}$ ).

Using the communication system, between the terminals, a very sensitive system can be designed. If the communication is partially or totally lost, actions can still be taken based on local criteria. Different load shedding steps that take the power system response into account, in order not to overshed, can easily be designed.

Protection systems against voltage instability can use simple binary signals such as "low voltage" or more advanced indicators such as power transfer margins based on the VIP algorithm [3] or modal analysis.

The solution with interconnected system protection terminals for future transmission system applications is illustrated in Fig. 1 for protection against voltage instability; similar illustration can be done for angular instability.

More details about the "flat architecture" are given in [4].

\section{Multilayered Architecture}

While the above two designs attempt at extending the "reach" of existing control domains (protection terminal being one domain and EMS being the other), there is no guarantee that the end solution will be comprehensive. A comprehensive solution is one that integrates the two control domains, protection devices and EMS. Such a solution is depicted in Fig. 2.

There are up to three layers in this architecture. The bottom layer is made up of PMUs, or PMUs with additional protection functionality. The next layer up consists of several local protection centers (LPCs), each of which interfaces directly with a number of PMUs. The top layer, the system protection center (SPC), acts as the coordinator for the LPCs.
Designing the three-layered architecture can take place in several steps. The first step should aim at achieving the monitoring capability, e.g., a WAMS. WAMS is the most common application based on PMUs. These systems are most frequent in North America, but are emerging all around the world. The main purpose is to improve state estimation, postfault analysis, and operator information. In WAMS applications, a number of PMUs are connected to a data concentrator, which basically is a mass storage, accessible from the control center, according to Fig. 3.

Starting from a WAMS design, a data concentrator can be turned into a hub-based local protection center (LPC) by implementing control and protection functions in the data concentrator (Fig. 4).

A number of such local protection centers can then be integrated into a larger system wide solution with an SPC at the top; see Fig. 2. With this solution the local protection center forms a system protection scheme (SPS), while the interconnected coordinated system forms a defense plan [5], [6].

\section{INSTITUTIONAL TRENDS}

As the electricity market is restructured all around the world, the nature of utility companies is changed. In particular, the downsizing of staff makes it difficult for the company to perform many R\&D functions. As a result, there is a trend in the industry where utility companies collaborate with vendors. The utility company can view its partnering vendor as a substitute for its vanishing $R \& D$ department to perform tasks that its existing staff cannot handle. The vendor sees the partnering utility as the "sounding board" for its product development and the place to demonstrate its latest products. This closed-loop collaboration, which already exists in the form of pilot projects in wide-area protection, is found to be fruitful to both parties.

\section{DISCUSSION}

The meaning of wide-area protection, emergency control, and power system optimization may vary dependent on 
people, utility, and part of the world, although the basic phenomena to be resolved are the same. Therefore, standardized and accepted terminology is important.

The solution to counteract the same physical phenomenon might vary extensively for different applications and utility conditions. A certain utility might wish to introduce a complete system to take care of a large number of applications, while others want to start with small installations of new technology in parallel with present systems. Some utilities want to do large amount of the studies, design, and engineering themselves, while others want to buy complete turnkey systems. It is important for any vendor in this area to supply solutions that fit with different utility organizations and traditions.

The potential to improve power system performance using smart control, as a complement to high-voltage equipment installations, seems to be great for many power grids.

\section{CONCLUSION}

This paper has discussed terminology, phenomena, and solution implementation strategies for wide-area protection. It is concluded that different applications will require different solutions. Therefore, system design and equipment must be very flexible both in size and complexity.

The use of the PMU has greatly improved the observability of the power system dynamics. Based on the PMU's different kinds of wide-area protection, emergency control and optimization systems can be designed.

A great deal of engineering, such as power system studies and configuration and parameter settings, is required, since every wide-area protection installation is unique. A cost effective solution could be based on standard products and standard system designs.

\section{REFERENCES}

[1] EPRI/DoD complex interactive networks and systems initiative: Self-healing infrastructures. [Online]. Available: http://www.epri. com/attachments/231 875_CINSIGOP_BAC_Feb_2000.PDF

[2] M. Amin, "Toward self-healing energy infrastructure systems," IEEE Comput. App. Power, vol. 14, no. 1, p. 20, Jan. 2001.

[3] W. H. Quaintance et al., "Raising energy transfer in corridors constrained by voltage instability-statnett case," in IEEE Power Engineering Soc. Summer Meeting 2000, vol. 4, pp. 2021-2026.

[4] P.-A. Löf, "New principles for system protection schemes in electric power networks," presented at the Bulk Power System Dynamics and Control V, Onomichi, Japan, 2001.

[5] "System protection schemes in power networks," CIGRE, Paris, France, TF 38.02.19, 2001

[6] C. Rehtanz, "Online stability assessment and wide area protection based on phasor measurements," presented at the Bulk Power System Dynamics and Control V, Onomichi, Japan, 2001.

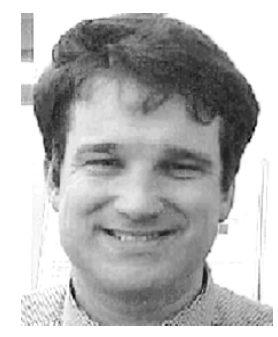

protection systems.

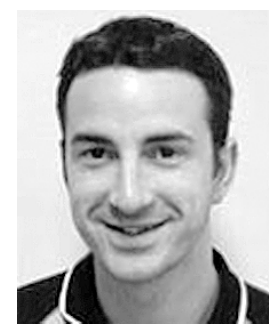

control system for $\mathrm{ABB}$.

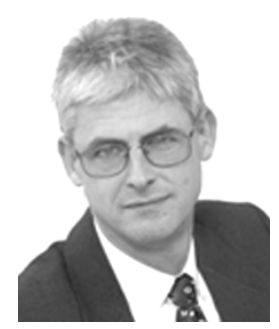

Daniel Karlsson (Senior Member, IEEE) received the Ph.D. degree in electrical engineering from Chalmers University in 1992.

Between 1985 and 1999, he was an Analysis Engineer at the Power System Analysis Group within the Operation Department of the Sydkraft utility. From 1994 until he left Sydkraft in 1999, he was Power System Expert and promoted to Chief Engineer. His work has been in the protection and power system analysis area and the research has been on voltage stability and collapse phenomena with emphasis on the influence of loads, on-load tap-changers, and generator reactive power limitations. His work has comprised theoretical investigations at academic level, as well as extensive field measurements in power systems. He is currently Application Senior Specialist at ABB Automation Technology Products.

Dr. Karlsson is a Member of CIGRE. Through the years he has been active in several Cigré and IEEE working groups. He has also supervised a number of diploma workers and Ph.D. students at Swedish universities.

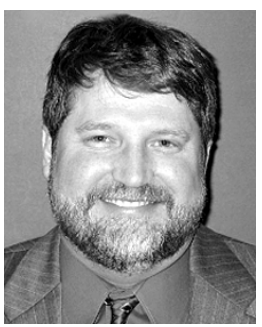

John McDaniel received the B.S.E.E. degree from Case Western Reserve University, Cleveland, $\mathrm{OH}$, and performed graduate studies in computer science at Southern Methodist University, Dallas, TX.

He has been with ABB, Sugar Land, TX, for over 19 years, working in the Network Management division responsible for supervisory control and data acquisition (SCADA) and energy management system (EMS) development. As Director of Engineering for the past ten years, John has been responsible for system architecture of the SCADA/EMS product and for all customer projects. In this role John has also been involved in research projects related to SCADA/EMS, including the Phasor Measurement Unit (PMU) research project.

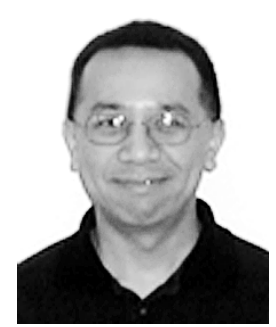

Khoi Vu received the Ph.D. degree in electrical engineering from the University of Washington, Seattle, and the MBA degree from the University of North Carolina, Chapel Hill.

He has been with ABB Corporate Research, Raleigh, NC, since 1993 and works in wide-area protection, protective-relay algorithms, power quality, energy markets, demand response, and power-system analysis. 